\title{
Efficient Cross-validation in ILP
}

\author{
Jan Struyf and Hendrik Blockeel \\ Katholieke Universiteit Leuven, Dept. of Computer Science, \\ Celestijnenlaan 200A, B-3001 Leuven, Belgium \\ \{Jan.Struyf, Hendrik.Blockeel\}@cs.kuleuven.ac.be
}

\begin{abstract}
Cross-validation is a technique used in many different machine learning approaches. Straightforward implementation of this technique has the disadvantage of causing computational overhead. However, it has been shown that this overhead often consists of redundant computations, which can be avoided by performing all folds of the crossvalidation in parallel. In this paper we study to what extent such a parallel algorithm is also useful in ILP. We discuss two issues: a) the existence of dependencies between parts of a query that limit the obtainable efficiency improvements and b) the combination of parallel cross-validation with query-packs. Tentative solutions are proposed and evaluated experimentally.
\end{abstract}

\section{Introduction}

Cross-validation is a technique used in many different machine learning approaches, such as instance based learning, artificial neural networks or decision tree induction, to tune parameters, select relevant features or to estimate predictive accuracies. Running an $n$-fold cross-validation consists of partitioning the data set $D$ into $n$ subsets $D_{i}$ and then running the given learning algorithm $n$ times, each time using a different training set $T_{i}=D-D_{i}$ and a different validation set $D_{i}$.

An obvious disadvantage of performing cross-validation is the computational overhead of running the learning algorithm $n$ times. This is a problem for ILP systems which are known to have high execution times. However, for some systems, this overhead can be reduced significantly. The training sets $T_{i}$ are highly similar. Indeed, each example from the original data set $D$ occurs $n-1$ times as training example. Because of this similarity, redundant computations are performed in the different cross-validation runs. These redundancies can be removed by integrating the different runs into one single algorithm. This is shown in [4] for decision tree induction.

Similarities between the training sets are not the only kind of similarities that can cause an ILP system to do redundant computations. ILP systems typically search a large hypothesis space. This involves testing a huge number of first order logic queries on the training set. Most ILP algorithms search through the hypothesis space in a greedy manner. First order decision tree learners (TILDE [1], S-Cart [8]) for example consider refinements of the query from the previous 
level of the tree when selecting a query for a new node. A refinement of a query is obtained by extending it with new literals. This means that different refinements of the same query are highly similar (share literals). One can imagine that there will be redundant computations when testing these similar queries separately on the training set. It is shown in [2] that this kind of redundancies can be removed by integrating the similar queries in one so-called query-pack.

A first goal of this text is to discuss efficient cross-validation from an ILP point of view. We use decision tree induction to explain the concepts, but the method for efficient cross-validation can also be integrated in rule induction systems like FOIL [11] or Progol [9]. We discuss an important problem related to the fact that a query in a given node of a first order decision tree depends on the queries of higher level nodes. This query-dependency problem also occurs to some extent for rule induction. We show how the parallel cross-validation algorithm from [4] can be adapted to reduce the overhead caused by this problem. A second goal of this text is to investigate how the query-packs from [2] can be integrated in the parallel cross-validation algorithm.

This paper is organised as follows. Section 2 summarises logical decision tree induction, efficient decision tree cross-validation and query-packs. Section 3 discusses the query-dependency problem, shows how query-packs can be integrated in the parallel algorithm and suggests how the parallel algorithm can be modified for rule induction. Section 4 presents experimental results. We investigate the possible efficiency gain of combining parallel cross-validation with query-packs and the effect of the query-dependency problem. Section 5 states the conclusions.

\section{Preliminaries}

\subsection{Logical Decision Tree Induction}

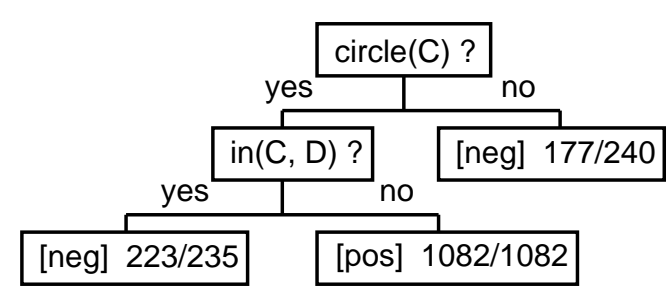

Fig. 1. A first order decision tree.

A first order decision tree [1] is a binary decision tree with conjunctions of first order literals in the nodes. The leaves contain class values in case of a classification task or (vectors of) real values in case of a regression task. An example tree grown on one of the Bongard data sets [6] is shown in Fig. 1. The 
prediction task for this set is classifying pictures containing circles, squares and triangles as positive or negative. We use the learning from interpretations setting [5] in which each example is given by a set of (Prolog) facts. Notice that it is not necessary to include a key variable in this setting.

First order decision trees are grown top down. The induction algorithm starts with the trivial query true. It then continues to add new nodes to the tree until a stop criterion is satisfied. The literal for a new node is selected by a greedy algorithm. It first generates refinements of the current query by extending it with new literals. In the Bongard example possible refinements of the query circle(C) are (circle(C), triangle(D)), (circle(C), square(D)), ( $\operatorname{circle}(C)$, in (C,D)), .. The algorithm computes a quality measure such as information gain [10] for each refinement. The refinement that maximises this quality is used to create the new node.

\subsection{The Parallel Algorithm}

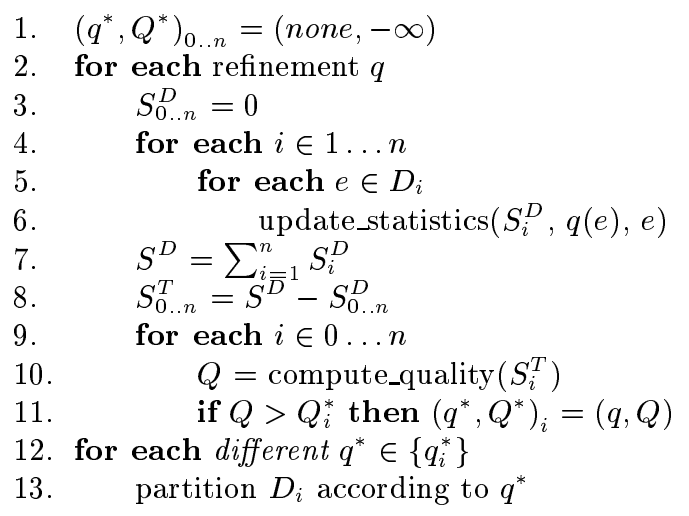

Fig. 2. Parallel cross-validation.

As explained briefly in the introduction, decision tree cross-validation involves growing $n$ different trees, each on a slightly different training set $T_{i}=$ $D-D_{i}$. Because the training sets $T_{i}$ are highly similar, one can expect that the trees will be highly similar too, especially near the root. The parallel algorithm [4] shown in Fig. 2 exploits this similarity while growing $n+1$ different trees at once. One tree for each cross-validation fold and one tree grown on the whole data set $D=T_{0}$. We call this set of $n+1$ trees the cross-validation forest. The algorithm keeps track of a tuple $\left(q^{*}, Q^{*}\right)_{i}$ (Line 1) for each tree of the forest. The first component of this tuple, $q^{*}$, is the best query found so far and the second component, $Q^{*}$, is $q^{*}$ 's quality. In each iteration another refinement $q$ is evaluated on the data (Lines $3-10)$. Line 11 updates $\left(q^{*}, Q^{*}\right)_{i}$ if $q$ is better than $q_{i}^{*}$. 
Lines 3 - 6 compute statistics $S_{i}^{D}$ for query $q$ on each set $D_{i}$ of the crossvalidation partition. Because these sets are disjoint, the query is evaluated only once on each example from $D$. To estimate the quality $Q$ (Line 10) we need statistics $S_{i}^{T}$ on the training sets $T_{i}$, which are derived from the previously calculated $S_{i}^{D}$ (Line 7 - 8). This step is only possible if the statistics are additive, meaning that $S^{A_{1} \cup A_{2}}=S^{A_{1}}+S^{A_{2}}$ if $A_{1} \cap A_{2}=\emptyset$. Each statistic $S$ is a tuple with two components $P S$ and $N S$. The positive component $P S$ is updated if $q(e)$ succeeds (Line 6$)$ and the negative component $N S$ is updated if $q(e)$ fails.

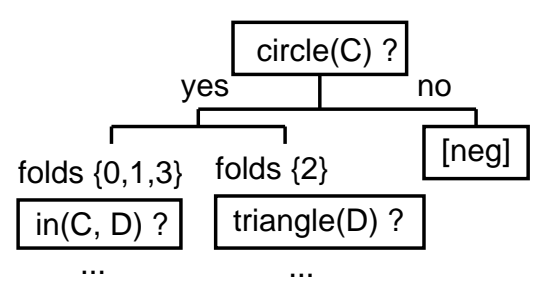

Fig. 3. A 3-fold cross-validation forest.

The last two lines of the algorithm update the nodes of the forest. A forest is not really a set of disjoint trees, there is some amount of sharing between the trees. The trees continue to share nodes as long as the same query $q^{*}$ is selected for each tree. A group of trees or a single tree can be split off from the forest if the algorithm selects a different $q^{*}$ for this group. An example forest is shown in Fig 3. At level two the tree for training set $T_{2}$ is split off from the forest and stops sharing computations.

It is shown in [4] that the speedup factor $T_{\text {serial }} / T_{\text {parallel }}$ is given by $\left(t_{\mathrm{r}}(i)\right.$ is the average time for growing one level of a single tree from $D$ and $f(i)$ is the average number of tree-groups that have been split off in the forest):

$$
\frac{n \cdot t_{\mathrm{r}}(1)+n \cdot t_{\mathrm{r}}(2)+n \cdot t_{\mathrm{r}}(3)+\ldots}{t_{\mathrm{r}}(1)+f(1) \cdot t_{\mathrm{r}}(2)+f(2) \cdot t_{\mathrm{r}}(3)+\ldots}
$$

Because tree-groups usually split off at lower levels of the forest, where only a few examples are left, speedup is in most cases quite good.

\subsection{Query-packs}

Figure 4 shows refinements for the query circle(C), in (C,D). The parallel algorithm considers each of these refinements if it has to select the best query for the left - left subtree of the Bongard forest from Fig. 3. All these refinements have the first two literals in common and executing these queries separately on the training set will cause redundant computations. By integrating the queries in a query-pack [2] as shown in the right part of Fig. 4, this redundancy can be removed. 
A query-pack is a tree structure with literals or conjunctions of literals in the nodes. Each path from the root to some node represents a conjunctive query. Decision tree query-packs can be compared to brooms. The current query, its length being proportional to the current tree depth, forms the stick of the broom. It is shown in [2] that the speedup factor $T_{\text {sequential }} / T_{\text {pack }}$ ranges from 1 to $\min (c+1, b)$ where $b$ is the branching factor of the pack and $c$ is the ratio of the computational complexity in the shared part over the complexity in the non-shared part. Because a broom has a long shared part and a high branching factor, one can expect high speedups.
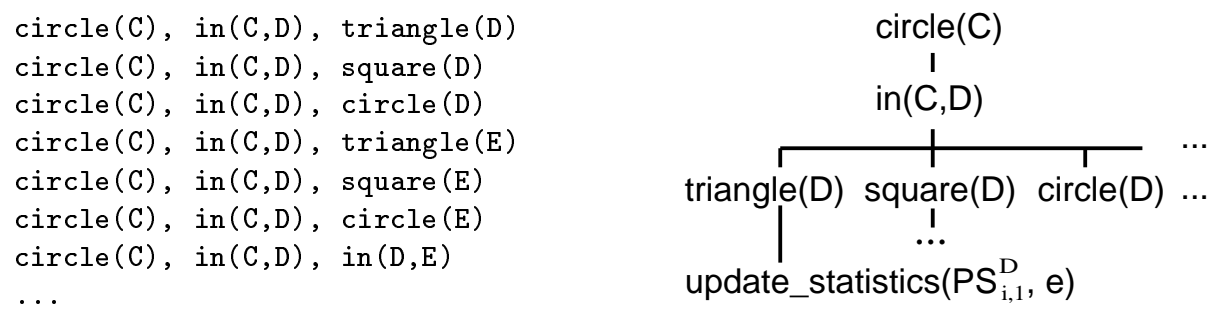

Fig. 4. A query-pack.

Note the similarity between a query-pack and a cross-validation forest. The structure and the basic idea are the same but the goals are different. A crossvalidation forest represents the shared part of similar decision trees and a querypack represents the shared part of similar queries.

\section{Parallel Cross-validation in ILP}

In this section we introduce the query-dependency problem and show how the query-packs from the previous section can be integrated in the parallel crossvalidation algorithm. We discuss the latter in the context of first order rule and constraint induction systems.

\subsection{The query-dependency problem}

In the parallel algorithm from [4] sharing of computations stops once a group of folds is split off from the main forest. In the propositional case, it is relatively easy to share computations between different fold groups. For ILP systems this is more difficult because of the query-dependency problem. In this section we discuss this query-dependency problem and show how the parallel algorithm can be improved to allow sharing of computations among different fold groups.

Consider again the example forest from Fig. 3. Let $G^{1}$ be the group for folds $\{0,1,3\}$ and $G^{2}$ the group for fold $\{2\}$. The best query for $G^{1}, q^{1}=(\operatorname{circle}(\mathrm{C})$, in (C, D) ), differs from the best query for $G^{2}, q^{2}=(\operatorname{circle}(C)$, triangle(D)). 
Although $q^{1}$ and $q^{2}$ are different it is likely that the partitions $\left(D^{+}, D^{-}\right)^{1}$ and $\left(D^{+}, D^{-}\right)^{2}$ they induce have a large overlap $\left(\left|D^{1+} \cap D^{2+}\right| \gg 0\right)$. This is because $q^{1}$ and $q^{2}$ both are best queries on a similar data set.

Suppose that the partitions are similar. We now move to the next level of the forest and evaluate all refinements of $q^{1}$ on $D^{1+}$ and all refinements of $q^{2}$ on $D^{2+}$. This does not involve redundant computations because the refinements of $q^{1}$ are all different from the refinements of $q^{2}$. This is because the refinements of $q^{1}$ contain the literal in (C,D) and the refinements of $q^{2}$ contain the literal triangle(D). This effect, to which we referred in the introduction as the querydependency problem, does not occur for a propositional decision tree learner because this kind of system does not have variables that link tests from different levels of the tree.

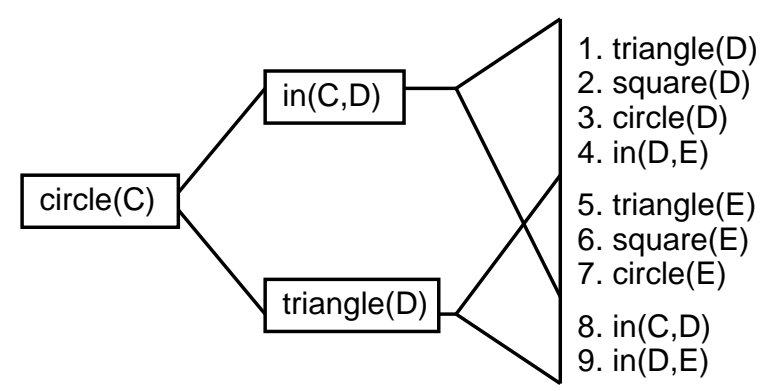

Fig. 5. Overlapping refinements.

Figure 5 shows all refinements for $q^{1}$ and $q^{2}$. Each refinement is a conjunction of 3 literals. The first literal is always circle(C), the second either in (C,D) or triangle(D). For the last literal we have three different cases: it occurs only in $q^{1}$ 's refinement set (literals $1-4$ in Fig. 5), it occurs only in $q^{2}$ 's refinement set $(8-9)$ or it occurs in both sets $(5-7)$. The last case is of course the most interesting. Is it possible to remove the second literal and gain efficiency by evaluating refinements $5-7$ only once instead of twice on the data?

Smartcall [3] is a query transformation that removes literals from a clause which are known to succeed. It first partitions the query in equivalence classes. Two literals are in the same class if they share (indirectly) variables. Refinement 5 of $q^{1}$ for example, can be partitioned in two classes $C_{1}=\{\operatorname{circle}(\mathrm{C})$, in (C,D) $\}$ and $C_{2}=\{$ triangle(E) $\}$. The literals in $C_{1}$ are known to succeed because they were used to partition the data at a higher level of the tree. We remove $C_{1}$ from the query and obtain triangle(E).

Smartcall removes the first two literals of refinements $5-7$ for both $q^{1}$ and $q^{2}$. This is shown on the left side of Fig. 6 . The rectangle represents the overlap of the refinement sets in the vertical dimension and the overlap of the example sets in the horizontal dimension. If the overlap is big in both dimensions then we 
can expect a high speedup by evaluating overlapping queries only once on the intersection of the example sets. Remember that the overlap of the example sets is large if similar queries are selected in a higher node of the tree. The overlap in the refinement sets is large if Smartcall can remove the crucial literals. Notice that propositional tests can always be removed because they do not introduce new variables.

Although we have considered only two groups $G^{1}$ and $G^{2}$, everything said in this section can be generalised to $k>2$ groups. One problem is that the intersection of the refinement sets and the intersection of the example sets is smaller. We solve this by adding groups using a greedy algorithm until the number of examples in the intersection drops below a given threshold.

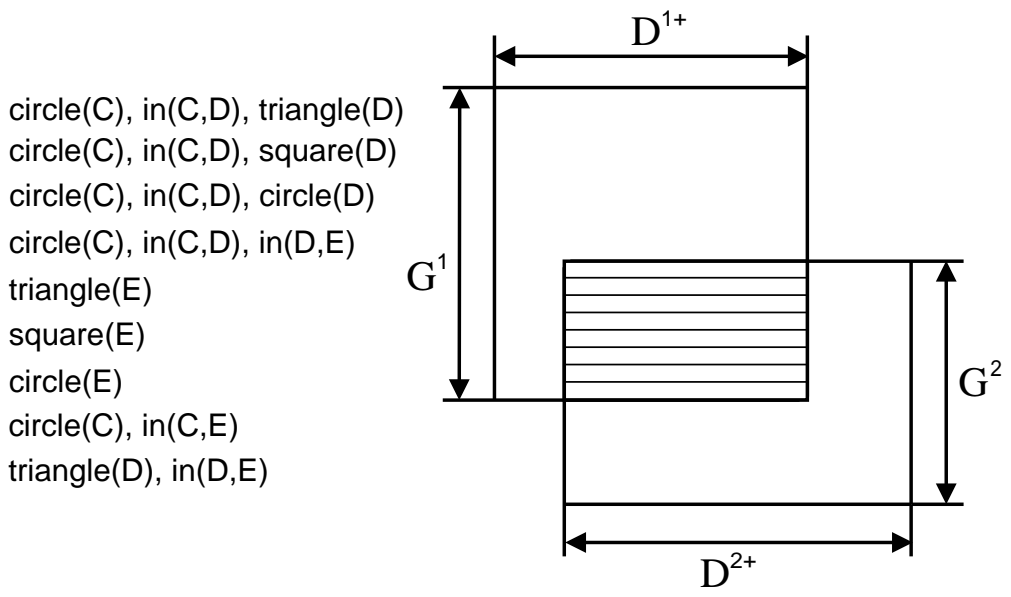

Fig. 6. Overlapping refinements and data sets.

Another problem we did not discuss so far is the problem of anti-similar queries. Suppose that we have a classification task with two classes pos and neg. If query $q_{1}$ moves almost all positive examples to the left and query $q_{2}$ moves almost all positive examples to the right then we have anti-similar queries. The extreme case is that $q_{1}=\neg q_{2}$. This problem can be solved by making sure that the left subtree always covers the most positive examples. We swap the left and right subtree if this is not the case. A similar approach can be used for regression (put the set with the lowest mean on the left) or for classification problems with more than two classes (impose an artificial order on the classes and put the set with the smallest most frequent class on the left).

\subsection{Combination with Query-packs}

Figure 7 shows the parallel cross-validation algorithm adapted to use querypacks. Line 3 of the adapted algorithm creates the pack $\mathcal{Q}$ and returns the 
number of queries in the pack (i.e. the pack size) $s$. Lines $4-6$ evaluate the pack on the data. Because the query-pack integrates $s$ different refinements, we have to keep track of a $(n+1) \times s$ statistic matrix. The rows of this matrix correspond to the different trees in the forest and the columns correspond to the different queries in the pack.

1. $\left(q^{*}, Q^{*}\right)_{0 . . n}=($ none,$-\infty)$

2. refine_and_compile_pack $(\mathcal{Q}, s)$

3. $P S_{0 . . n, 1 . . s}^{D}=0$

4. for each $i \in 1 \ldots n$

5. for each $e \in D_{i}$

6. $\quad$ execute_pack $\left(\mathcal{Q}, P S_{i}^{D}, e\right)$

7. $P S^{D}=\sum_{i=1}^{n} P S_{i}^{D}$

8. $P S_{0 . . n}^{T}=P S^{D}-P S_{0 . . n}^{D}$

9. for each $j \in 1 \ldots s$

$10 q=$ get_from_pack $(\mathcal{Q}, j)$

11. for each $i \in 0 \ldots n$

12. $N S_{i, j}^{T}=T S_{i}^{T}-P S_{i, j}^{T}$

13. $Q=$ compute_quality $\left(T S_{i}^{T}, P S_{i, j}^{T}, N S_{i, j}^{T}\right)$

14. $\quad$ if $Q>Q_{i}^{*}$ then $\left(q^{*}, Q^{*}\right)_{i}=(q, Q)$

15. for each different $q^{*} \in\left\{q_{i}^{*}\right\}$

16. partition $D_{i}$ according to $q^{*}$

Fig. 7. Packs version of the parallel algorithm.

The leaves of the pack contain update_statistic functions (See Fig. 4). These functions update the positive component $P S_{i, j}^{D}$ of the statistics $S_{i, j}^{D}$ for each query $q_{j}$ in the pack. Updating the negative component of $S_{i, j}^{D}$ is not possible with the pack representation discussed in Section 2.3. However, $N S_{i, j}^{T}$ can be calculated indirectly, by introducing total statistics $T S_{i}^{D}$ and $T S_{i}^{T}$. Total statistics are equal for all refinements $q_{j}$ and can be computed from the data before the parallel algorithm is started. The negative component $N S_{i, j}^{T}$ can be derived using the equality $T S_{i}^{T}=P S_{i, j}^{T}+N S_{i, j}^{T}$ (Line 12). The rest of algorithm is similar to the version without packs (See Fig. 2).

\subsection{Rule induction}

Although we focused on decision tree induction, almost everything said so far also applies to top down rule induction (e.g. FOIL [11], Progol [9]) and to top down constraint induction (e.g. ICL[7]). A top down rule induction system tries to cover all positive examples by learning a disjunction of conjunctive rules. Each time a new rule is learned, the systems removes the covered positive examples from the data set and tries to learn a next rule until all positive examples are covered or no more good rules can be found. To learn one rule, the system starts 
from the most general rule true and keeps adding the best literal according to some quality measure as long as the rule's quality improves.

We can use the ideas from the parallel decision tree cross-validation algorithm to build a parallel rule cross-validation algorithm. This parallel rule cross-validation algorithm builds a tree that represents the shared part between rules for different folds. If one or more folds select a different literal then the rule for this group of folds is split off from the main tree and forms a new branch. This can be compared to the cross-validation forest from Section 2.2. As long as a group of folds remains together, computations can be shared by evaluating the rule for this group on the disjoint sets $D_{i}$ and not on the overlapping training sets $T_{i}$.

If it is possible to use the Smartcall query transformation (i.e. if the positive examples that are not covered by the current rule are removed each time a new literal is added) then it is also possible to share computations between different branches of the rule tree in a similar way as discussed in Section 3.1.

Before a rule induction system starts to learn a new rule, it removes all positive examples covered by the previous rule. As long as the same rules are selected for different folds of the cross-validation (i.e. the tree has no branches) the same examples are removed from the data set $D$ and $D$ remains equal for all folds. If fold $i$ selects a different rule then different examples will be removed from $D$ and the data set for fold $i$ will differ from the data sets of the other folds. If we partition $D$ in $\left(D^{s}, D^{1} \ldots D^{n}\right)$, where $D^{s}$ contains the shared examples and $D^{i}$ contains the examples for fold $i$ not in $D^{s}$, then it is possible to share computations over $D^{s}$.

Progol uses one of the positive examples $e$ to constrain its hypothesis space $H$ to a space $H_{e}$ which only contains hypotheses more general than $e$. After that, it performs an exhaustive search in $H_{e}$ looking for the rule that maximises a quality measure called compaction. When running an n-fold cross-validation, each fold will select a different example $e_{i}$ but some of the hypothesis spaces $H_{e_{i}}$ will be equal. This means it is possible to remove redundancies among folds that share the same $H_{e}$.

\section{Experimental Results}

For our experiments we implemented the different cross-validation optimisations discussed in this text as a module of TILDE, the first order decision tree learner from the ACE data mining tool ${ }^{1}[2]$.

We compare execution times of a 10-fold run for serial (no optimisations), serial + query-packs, parallel, parallel + intersection (share computations among different groups of trees) and finally parallel cross-validation + query-packs. The data sets used are:

- The simple (SB) and complex (CB) Bongard data set [6] (this set was also used as running example in this text). SB contains 1453 examples with a

\footnotetext{
${ }^{1} \mathrm{ACE}$ is available for academic purposes upon request. http://www.cs.kuleuven.ac.be/ dtai/ACE/
} 
simple underlying theory, CB contains 1521 examples with a more complex theory.

- A subset (ASM) of 999 examples sampled from the "Adaptive Systems Management" data set, kindly provided to us by Perot Systems Nederland.

- The Mutagenesis (Muta) data set [12], an ILP benchmark (230 examples).

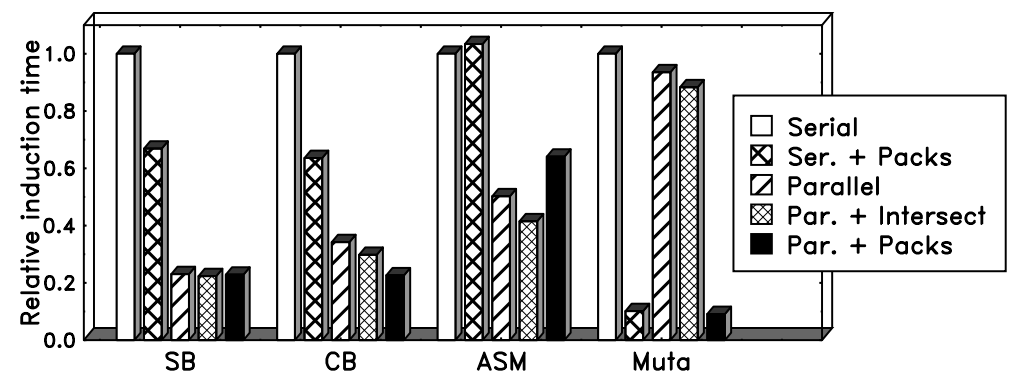

Fig. 8. Different cross-validation optimisations (time relative to serial 10-fold).

As can be seen in Figure 8, Table 1 and Table $2,{ }^{2}$ the results are not uniform for all data sets. This is because we deliberately selected different types of data sets. For SB all parallel algorithms perform more or less the same. The intersection algorithm does not perform better because no groups are split off from the forest. The packs version does not perform better because the queries are too short to have many literals in common. CB is the least surprising data set: some groups are split off from the forest and the queries become longer. The ASM data set contains many propositional numeric attributes. The combination of the parallel algorithm with intersection performs well on this data set because it has a lot of propositional attributes that can be removed by Smartcall. One problem is that intersecting refinement sets (using a hash tree) and example sets (sorted lists) is still rather slow in our implementation. Query-packs perform very bad on ASM because the pack has a high branching factor (it contains a huge number of tests comparing numeric attributes to each of their discretised values). The time necessary for compiling a pack depends on the pack size. Near the leaves this compilation time dominates the execution time which is linear in the number of examples. The Mutagenesis data set is not suited for parallel cross-validation because some queries, generated near the leaves of the forest, dominate the total execution time [4] (this happens when the algorithm looks for circular substructures in the molecules). Query-packs on the other hand perform very well for Mutagenesis (10 times faster). This is because query-pack execution shares computations among the different refinements of this few complex queries.

\footnotetext{
${ }^{2}$ The timings are faster comparing to [4] because we have ported the statistics code from Prolog to $\mathrm{C}++$.
} 
Table 1. Timings (seconds) comparing parallel to serial cross-validation (10-folds), once with packs disabled and a second time with packs enabled.

\begin{tabular}{|c|c|c|c|}
\hline \multirow{3}{*}{$\begin{array}{l}\text { SB } \\
\text { Serial } \\
\text { Parallel }\end{array}$} & \multirow{2}{*}{$\frac{\text { Packs off }}{16}$} & \multicolumn{2}{|c|}{ Packs on } \\
\hline & & 11 & $1.5 \times$ \\
\hline & 3.6 & 3.5 & $1.0 \times$ \\
\hline & $4.4 \times$ & $3.1 \times$ & \\
\hline \multicolumn{4}{|l|}{ CB } \\
\hline Serial & 24 & 15 & $1.6 \times$ \\
\hline Parallel & 8.2 & 5.4 & $1.5 \times$ \\
\hline & $2.9 \times$ & $2.8 \times$ & \\
\hline
\end{tabular}

\begin{tabular}{|c|c|c|c|}
\hline \multirow{3}{*}{$\begin{array}{l}\text { ASM } \\
\text { Serial } \\
\text { Parallel }\end{array}$} & \multirow{3}{*}{$\begin{array}{l}\text { Packs off } \\
4100 \\
2100\end{array}$} & \multicolumn{2}{|c|}{ Packs on } \\
\hline & & 4300 & $0.95 \times$ \\
\hline & & 2600 & $0.81 \times$ \\
\hline & $2.0 \times$ & $1.7 \times$ & \\
\hline \multicolumn{4}{|l|}{ Muta } \\
\hline Serial & 5000 & 500 & $10 \times$ \\
\hline Parallel & 4600 & 450 & $10 \times$ \\
\hline & $1.09 \times$ & $1.11 \times$ & \\
\hline
\end{tabular}

Table 2. The effect of sharing computations between different fold groups (10-folds, times in seconds).

\begin{tabular}{lcccc}
\hline & SB & CB & ASM & Muta \\
\hline Parallel & 3.6 & 8.2 & 2100 & 4600 \\
Intersect & 3.5 & 7.1 & 1710 & 4400 \\
\hline \multicolumn{4}{c}{$1.03 \times 1.15 \times 1.22 \times$} & $1.05 \times$ \\
\hline
\end{tabular}

\section{Conclusions}

We discussed two optimisations specific to ILP for the parallel decision tree crossvalidation algorithm proposed in [4]. The first one was intersecting refinement and example sets to be able to share computations between different fold groups of the cross-validation forest. The second one was integrating query-packs in the parallel algorithm.

One possible improvement is to implement the combination of the two optimisations discussed in this text: parallel cross-validation with query-packs and intersection. In order to implement this, one would need an equivalent to Smartcall for query-packs. This is future work.

It became clear from the experiments that different optimisations work well for different data sets. The more optimisations that are integrated in a learning system, the more difficult it is for an end-user to know which optimisations are suited for his particular data set. Maybe it is possible to use meta-learning to decide which optimisations have to be used for a given set.

We also discussed how the ideas from the parallel decision tree cross-validation algorithm can be used to devise a parallel rule or constraint induction cross-validation system. 


\section{Acknowledgements}

The authors are a research assistant, respectively post-doctoral fellow, of the Fund for Scientific Research of Flanders (Belgium). They thank Perot Systems Nederland / Syllogic for providing the ASM data. The cooperation between Perot Systems Nederland and the authors was supported by the European Union's Esprit Project 28623 (Aladin).

\section{References}

1. H. Blockeel and L. De Raedt. Top-down induction of first order logical decision trees. Artificial Intelligence, 101(1-2):285-297, June 1998.

2. H. Blockeel, B. Demoen, L. Dehaspe, G. Janssens, J. Ramon, and H. Vandecasteele. Executing query packs in ILP. In J. Cussens and A. Frisch, editors, Proceedings of the 10th International Conference in Inductive Logic Programming, volume 1866 of Lecture Notes in Artificial Intelligence, pages 60-77, London, UK, July 2000. Springer.

3. H. Blockeel, B. Demoen, G. Janssens, H. Vandecasteele, and W. Van Laer. Two advanced transformations for improving the efficiency of an ILP system. In 10th International Conference on Inductive Logic Programming, Work-in-Progress Reports, pages 43-59, London, UK, July 2000.

4. Hendrik Blockeel and Jan Struyf. Efficient algorithms for decision tree crossvalidation. In Proceedings of ICML-2001 - Eighteenth International Conference on Machine Learning. Morgan Kaufmann, 2001. To appear.

5. L. De Raedt and S. Džeroski. First order $j k$-clausal theories are PAC-learnable. Artificial Intelligence, 70:375-392, 1994.

6. L. De Raedt and W. Van Laer. Inductive constraint logic. In Klaus P. Jantke, Takeshi Shinohara, and Thomas Zeugmann, editors, Proceedings of the Sixth International Workshop on Algorithmic Learning Theory, volume 997 of Lecture Notes in Artificial Intelligence, pages 80-94. Springer-Verlag, 1995.

7. L. De Raedt and W. Van Laer. Inductive constraint logic. Unpublished, 1995.

8. Stefan Kramer. Structural regression trees. In Proceedings of the Thirteenth National Conference on Artificial Intelligence, pages 812-819, Cambridge/Menlo Park, 1996. AAAI Press/MIT Press.

9. S. Muggleton. Inverse entailment and Progol. New Generation Computing, Special issue on Inductive Logic Programming, 13(3-4):245-286, 1995.

10. J. Ross Quinlan. C4.5: Programs for Machine Learning. Morgan Kaufmann series in machine learning. Morgan Kaufmann, 1993.

11. J.R. Quinlan. Learning logical definitions from relations. Machine Learning, 5:239266, 1990 .

12. A. Srinivasan, S.H. Muggleton, M.J.E. Sternberg, and R.D. King. Theories for mutagenicity: A study in first-order and feature-based induction. Artificial Intelligence, 85(1,2), 1996. 